\title{
DESENVOLVIMENTO PSICOMOTOR EM CRIANÇAS PRÉ-TERMO E A TERMO NA IDADE ESCOLAR
}

\author{
PSYCHOMOTOR DEVELOPMENT OF PRETERM AND \\ FULL TERM CHILDREN AT SCHOOL-AGE
}

Cardoso AA; Magalhães LC; Barbosa VM. Desenvolvimento psicomotor em crianças prétermo e a termo na idade escolar. Rev Bras Crescimento Desenvolvimento Hum. 2011; 21(2): 210-219.

\section{RESUMO}

Fatores biológicos, como prematuridade e baixo peso ao nascimento, e fatores ambientais, como nível sócioeconômico, podem influenciar o desenvolvimento infantil. O objetivo deste estudo foi comparar o desenvolvimento psicomotor de três grupos de crianças em idade escolar: (1) pré-termo de famílias de baixa renda, (2) a termo de famílias de baixa renda, e (3) a termo de classe média. Participaram do estudo 120 crianças de 5 a 7 anos de idade, 40 em cada grupo. As crianças foram submetidas a avaliação de equilíbrio estático, tônus postural, habilidades perceptuais e viso-motoras. Foi observada diferença significativa de desempenho entre os grupos na maioria dos testes. Ao analisar o efeito isolado do nível sócioeconômico, observou-se efeito favorável à classe média nos itens de tônus postural e equilíbrio. Os resultados sugerem que o desenvolvimento de crianças prematuras deve ser acompanhado até pelo menos a idade escolar, principalmente daquelas em situação de desvantagem social, sendo importante incentivar a implantação de programas de acompanhamento longitudinal do desenvolvimento dentro do sistema público de saúde.

Palavras-chave: prematuridade; desvantagem social; desenvolvimento humano; desempenho psicomotor; coordenação visomotora.

1 Terapeuta Ocupacional. Doutoranda em Ciências da Reabilitação, UFMG. Professora Assistente do Departamento de Terapia Ocupacional, Universidade Federal do Paraná, Curitiba, PR, Brasil. E-mail: anaameliato@yahoo.com.br

2 Terapeuta Ocupacional. Doutora em Educação. Professora Titular do Departamento de Terapia Ocupacional, Universidade Federal de Minas Gerais, Belo Horizonte, MG, Brasil. E-mail: liviam@gcsnet.com.br

3 Terapeuta Ocupacional. Doutora em Estudos Sobre Deficiência. Líder do Grupo de Pediatria, Departamento de Terapia Ocupacional, Centro Médico da Universidade de Illinois, Chicago, USA. E-mail: vanessambarbosa@yahoo.com.br Correspondência para: Ana Amélia Cardoso - Rua Padre Camargo, $280-3^{\circ}$ andar - Bairro Alto da Glória CEP 80060-240, Curitiba/PR - Fone/fax: (41) 3360-7273. e-mail: anaameliato@yahoo.com.br 


\begin{abstract}
Biological risk factors, such as prematurity, low birth weight and environmental factors, such as social class, can influence children development. The aim of this study was to compare the psychomotor development in three groups of school-age children - (1) preterm from low income families, (2) full term from low income families and (3) full term from middle class. The participants were 120 children, aged 5 to 7 years old, 40 in each group. Children were tested on static balance, postural, perceptual and visual motor skills measures. There was significant difference between groups in the majority of the tests. When analyzing the isolated effect of social class, a favorable effect for middle class in the postural and balance measurements was observed. The results suggest that the development of preterm children, especially in those from socially disadvantaged backgrounds, should be followed up to at least school age. Therefore, it is important to incentive the implementation of developmental follow-up programs in the public health system.
\end{abstract}

Key words: prematurity; social class; human development; psychomotor performance; visuomotor coordination.

\section{INTRODUÇÃO}

A prematuridade está relacionada à maior frequência de distúrbios de desenvolvimento ${ }^{1-3}$, principalmente quando associada ao baixo peso ao nascimento ${ }^{4-11}$. Apesar de não existir consenso quanto às características específicas dos desvios neuropsicomotores e a extensão dos déficits apresentados a longo prazo por essas crianças ${ }^{2,7-9}$, parece que mesmo na ausência de sinais severos, como paralisia cerebral e retardo mental, número significativo de crianças com história de prematuridade vem apresentar sinais de distúrbio de aprendizagem, dificuldades de linguagem, problemas de comportamento, déficits na coordenação motora e percepção visoespacial na idade esco$\operatorname{lar}^{2,3,6,12-14}$.

Embora a prematuridade esteja associada a maior frequência de déficits perceptuais, motores, distúrbios da atenção e comportamento ${ }^{2,3,6}$, alguns autores afirmam que fatores ambientais, em especial o baixo nível socioeconômico, seriam decisivos na determinação do desfecho, principalmente com relação ao desenvolvimento $\operatorname{cognitivo~}^{12-15}$. Há evidências de que à medida que a criança pré-termo cresce, fatores ambientais teriam maior influência sobre o desenvolvimento que os sinais neonatais de risco biológico ${ }^{8,16}$, sendo que, como medida preventiva, recomenda-se o acompanhamento do desenvolvimento dessas crianças. ${ }^{2,6,7,13,17}$

Considerando que no Brasil grande número de crianças estão expostas a fatores de risco social, é importante documentar o desempenho de crianças pré-termo brasileiras. Carvalho, Linhares e Martinez ${ }^{15}$ comparando a história de desenvolvimento de 20 crianças pré-termo de 8 a 10 anos, com pares nascidos a termo, identificaram sinais de atraso na aquisição de comportamentos psicomotores no grupo pré-termo. Além disso, as crianças pré-termo apresentaram mais repetência escolar, problemas respiratórios, enurese noturna, recusa escolar, agitação, impaciência, inquietude e agarramento à mãe do que as do grupo controle.

Méio et. al ${ }^{18}$ observaram que a média do quociente de inteligência (QI) de pré-escolares nascidos pré-termo estava abaixo da faixa de normalidade. Resultado semelhante ao de Linhares et $\mathrm{a}^{19}$, que observaram maior frequência de problemas de compor- 
tamento e nível intelectual abaixo da média em crianças nascidas pré-termo

Magalhães et $\mathrm{al}^{20}$, em um primeiro estudo sobre impacto da prematuridade no desenvolvimento perceptual e motor, examinaram 22 crianças pré-termo em idade escolar com testes de coordenação visomotora ${ }^{21,22}$, equilíbrio e tônus postural ${ }^{21}$. Foi observado que $63 \%$ da amostra apresentaram desempenho perceptomotor inconsistente ou nitidamente abaixo do esperado para a idade. Além dos problemas específicos da prematuridade, essas crianças estavam expostas a ambientes empobrecidos $\mathrm{e}$ a condições, como a desnutrição e estresse familiar, que podem influenciar o desenvolvimento infanti1 ${ }^{15,17,19,23}$.

Em um segundo estudo ${ }^{24}$, o desempenho psicomotor de 35 crianças pré-termo foi comparado aos pares nascidos a termo, de mesma idade, sexo e nível socioeconômico (crianças de baixa renda). Os resultados indicaram vantagem significativa para as crianças a termo em 10 das 11 variáveis incluídas no estudo. Como esses dois estudos ${ }^{20,24}$ incluíram apenas crianças expostas a fatores de risco social, em uma segunda etapa, considerou-se relevante comparar os dados com o desempenho motor de crianças sem risco social ou biológico.

Procurando investigar se, além da prematuridade, o status socioeconômico também influencia o desempenho em provas perceptomotoras, o objetivo é comparar o desempenho psicomotor de três grupos de crianças, diferenciados pela idade gestacional e nível social.

\section{MÉTODO}

\section{PARTICIPANTES}

Participaram do estudo crianças de cinco a sete anos de idade, distribuídas em três grupos, como descrito a seguir.

a) Grupo I - pré-termo de baixa renda: 40 crianças nascidas no Hospital das Clínicas da UFMG com peso ao nascimento $\leq 1500 \mathrm{~g}$ e/ ou idade gestacional $\leq 34$ semanas, e acompanhadas no Ambulatório da Criança de Risco (ACRIAR/UFMG) até a idade escolar. Foram excluídas crianças com paralisia cerebral, retardo mental, doenças genéticas, deficiências sensoriais e outras doenças não associadas à prematuridade. Todas as crianças do Grupo I eram provenientes de famílias de baixa renda (entre um a três salários mínimos).

b) Grupo II - pré-termo de baixa renda: 40 crianças nascidas a termo (peso ao nascimento $>2500$ g e idade gestacional $>37$ semanas) e sem história de intercorrências neonatais, pareadas por idade e sexo às crianças do Grupo I. As crianças do Grupo II foram recrutadas em uma Escola Municipal da periferia da Grande Belo Horizonte, selecionada especificamente por servir a população de nível socioeconômico semelhante ao das crianças atendidas no ACRIAR.

c) Grupo III - a termo de classe média: 40 crianças nascidas a termo (peso ao nascimento $>2500 \mathrm{~g}$ e idade gestacional $>37$ semanas) e sem história de intercorrências neonatais, pareadas por idade e sexo às crianças do Grupo I. As crianças do Grupo III foram recrutadas em uma escola tradicional da rede privada de Belo Horizonte, com excelente estrutura física e voltada para educação de crianças de famílias de classe média.

\section{INSTRUMENTAÇÃO}

Foram aplicados os seguintes testes:

(a) Teste Gestálico de Bender ${ }^{22}$ : teste muito conhecido no Brasil e usado para avaliar a coordenação visomotora, que consiste na cópia de 9 figuras geométricas com escore de acordo com o número de erros cometidos pela criança. Quanto mais baixo o escore melhor a coordenação visomotora. $\mathrm{O}$ teste de Bender foi administrado e pontuado segundo as instruções de Koppitz ${ }^{22}$.

(b) Teste de Acuidade Motora (Motor Accuracy Test - MAC) de Ayres ${ }^{21}$ : avalia a coordenação visomotora, examinando a acuidade 
com que a criança traça em cima de uma linha preta, em formato de borboleta, que circunda a folha de teste. Faz-se a medida da distância que o traçado saiu da linha e do tempo gasto no traçado. São dados dois escores, um para acuidade (MAC-Acuidade) e outro para a combinação entre o tempo gasto na prova e a distância traçada fora da linha preta (MAC-Ajustado), porém, neste estudo utilizamos apenas o escore de acuidade (MAC-Acuidade). Quanto maior o escore, melhor o desempenho da criança.

(c) Equilíbrio Estático: por meio da prova de equilíbrio em um pé só, com os olhos abertos e fechados, com limite máximo de tempo de 15 segundos. Este teste foi aplicado segundo instruções de Ayres ${ }^{21}$.

(d) Tônus Postural: foram usadas as provas de prono-extensão e supino-flexão ${ }^{21}$, que avaliam a força ou habilidade para conseguir manter padrões globais de flexão e extensão contra a gravidade. $O$ desempenho é avaliado quantitativamente, cronometrando o tempo que a criança mantém a posição, até um máximo de 25 segundos, e qualitativamente, registrando a qualidade da postura mantida durante a prova. Os escores qualitativos variam de 1 (incapaz de manter postura) a 4 (postura harmoniosa e simétrica).

\section{PROCEDIMENTOS}

As crianças do Grupo I foram avaliadas no Ambulatório Bias Fortes/UFMG, onde funciona o ACRIAR. As crianças do Grupo II e III foram avaliadas nas respectivas escolas, em horários estipulados pelos professores, de forma a não interferir no desempenho escolar da criança. A avaliação foi individual e durou cerca de 40 minutos.

Durante a testagem buscou-se criar um ambiente descontraído e divertido para a criança, sendo que os testes foram aplicados na seguinte ordem: prono-extensão, equilíbrio em um pé só com os olhos abertos e fechados e supino-flexão. Em seguida passou-se à mesa, para administração dos testes MAC e Bender.
Todas as crianças foram testadas por bolsistas de Iniciação Científica, sendo que as examinadoras foram treinadas de acordo com as instruções de cada manual de teste. Antes do início da coleta, examinou-se a confiabilidade entre examinadores em cerca de $10 \%$ da amostra. Os resultados indicaram congruência de escores acima de 90\%, com índices de correlação (Pearson $r$ ) entre examinadores variando de 0,99 a 1,00. O estudo foi aprovado pelo Comitê de Ética da Universidade Federal de Minas Gerais - Parecer ETIC 457/04.

A análise dos dados foi realizada usando o programa estatístico SPSS, versão 13.0. Como alguns dos testes usados não foram estandardizados para a criança brasileira, neste estudo foram analisados apenas os escores brutos obtidos em cada teste.

Inicialmente foi feita uma análise descritiva, caracterizando as médias e desvio padrão para cada grupo. Em seguida foi aplicado o Teste de Normalidade de Shapiro-Wilk, que detectou que a maioria das variáveis não seguia distribuição normal. Portanto, optou-se pelo uso de testes não-paramétricos: para variáveis quantitativas, foi utilizado o Teste de Friedman para comparação de $k$ grupos dependentes, seguido do Teste de Wilcoxon para comparação de dois grupos dependentes, com correção de Bonferroni.

Para as variáveis qualitativas, foi utilizado Teste Qui-Quadrado, com correção pelo Teste Exato de Fisher, quando necessário. Para verificar o efeito isolado dos fatores idade gestacional e renda no desempenho, foi utilizado o teste não paramétrico de MannWhitney.

\section{RESULTADOS}

Na Tabela 1 é apresentada a caracterização da amostra. Os resultados de cada grupo, bem como as diferenças estatisticamente significativas entre eles nas variáveis 
quantitativas, são apresentados na Tabela 2.

$\mathrm{O}$ Teste de Friedman não verificou diferen-

ça significativa entre os três grupos nos testes: MAC acuidade $(\mathrm{p}=0,962)$; equilíbrio OF-D $(\mathrm{p}=0,1234)$; equilíbrio OF-E $(\mathrm{p}=0,571)$.

Tabela 1: Caracterização dos participantes dos grupos I, II e III, em relação ao sexo e idade

\begin{tabular}{lccc}
\hline Participantes & \multicolumn{2}{c}{ Sexo } & Idade média \\
& Feminino & Masculino & em meses \\
\hline Grupo I(Pré-termo, baixa renda) & 14 & 26 & $75 \pm 6(65-91)$ \\
Grupo II(A termo, baixa renda) & 14 & 26 & $75 \pm 6(63-90)$ \\
Grupo III(A termo, classe média) & 14 & 26 & $75 \pm 6(63-92)$ \\
\hline
\end{tabular}

Tabela 2: Diferença de desempenho nos testes Bender, MAC, provas de equilíbrio e postura, entre os grupos I - pré-termo, baixa renda; II - a termo, baixa renda; e III - a termo, classe média.

\begin{tabular}{|c|c|c|c|c|c|c|}
\hline \multirow[t]{2}{*}{ Avaliação } & \multicolumn{2}{|c|}{$\begin{array}{l}\text { Grupo } 1 \\
\text { Pré-termo, baixa renda }\end{array}$} & \multicolumn{2}{|c|}{$\begin{array}{c}\text { Grupo } 2 \\
\text { A termo, baixa renda }\end{array}$} & \multicolumn{2}{|c|}{$\begin{array}{c}\text { Grupo } 3 \\
\text { A termo, classe média }\end{array}$} \\
\hline & Média \pm DP & amplitude & Média \pm DP & amplitude & Média \pm DP & amplitude \\
\hline Bender* & $11,68 \pm 4,55^{\mathrm{a}, \mathrm{b}}$ & $3-20$ & $8,05 \pm 3,37^{\text {a }}$ & $0-16$ & $9,13 \pm 3,78^{b}$ & $2-21$ \\
\hline MAC & $159,80 \pm 11,90$ & $122,00-193,37$ & $162,24 \pm 5,70$ & $150,50-178,00$ & $163,12 \pm 5,36$ & $151,70-172,70$ \\
\hline Eq. Olhos Abertos D & $10,85 \pm 5,05^{\mathrm{b}}$ & $1-15$ & $11,06 \pm 4,87^{\mathrm{c}}$ & $2-15$ & $14,38 \pm 1,31^{\mathrm{b}, \mathrm{c}}$ & $10-15$ \\
\hline Eq. Olhos Abertos E & $10,55 \pm 4,88^{b}$ & $2-15$ & $11,36 \pm 4,50^{\mathrm{c}}$ & $2,5-15$ & $13,73 \pm 2,29^{b, c}$ & $8-15$ \\
\hline Eq. Olhos Fechados D & $5,29 \pm 4,30$ & $1-15$ & $5,03 \pm 4,14$ & $0-15$ & $6,65 \pm 4,23$ & $2-15$ \\
\hline Eq. Olhos Fechados E & $4,81 \pm 3,83$ & $1-15$ & $5,01 \pm 3,78$ & $0-15$ & $6,25 \pm 3,69$ & $1-15$ \\
\hline Prono-extensão tempo & $16,28 \pm 8,11^{\mathrm{b}}$ & $0-25$ & $19,04 \pm 7,68^{\mathrm{c}}$ & $0-25$ & $23,95 \pm 3,88^{b, c}$ & $2-25$ \\
\hline Supino-flexão tempo & $15,73 \pm 8,26^{\mathrm{a}, \mathrm{b}}$ & $2-25$ & $19,51 \pm 7,60^{\mathrm{a}, \mathrm{c}}$ & $2,5-25$ & $23,18 \pm 4,05^{b, c}$ & $8-25$ \\
\hline
\end{tabular}

Nota: * Quanto maior o escore, pior o desempenho da criança; ${ }^{\text {a }}$ diferença significativa entre os grupos I e II; ${ }^{\mathrm{b}}$ diferença significativa entre os grupos I e III; ${ }^{c}$ diferença significativa entre os grupos II e III (testes de Friedman e de Wilcoxon, $\mathrm{p}<0,05$ )

Considerando-se as variáveis qualitativas, o teste qui-quadrado aponta associação significativa entre o desempenho e o grupo $(\mathrm{p}=0,004)$, no teste prono-extensão escore, com pior desempenho para o Grupo I.

Ao analisar o efeito isolado da idade gestacional (Tabela 3), o teste de MannWhitney aponta efeito significativo para os testes: Bender $(p=0,000)$; equilíbrio olhos abertos - perna esquerda $(p=0,031)$; supino-flexão tempo $(\mathrm{p}=0,000)$; prono-extensão tempo $(\mathrm{p}=0,004)$ e prono-extensão escore $(p=0,000)$, com melhor desempenho para crianças a termo.

Em relação ao nível socioeconômico (Tabela 4), observa-se efeito significativo para os testes: equilíbrio olhos abertos - perna direita $(\mathrm{p}=0,000)$; equilíbrio olhos abertos - perna esquerda ( $p=0,001)$; equilíbrio olhos fechados - perna direita $(p=0,021)$; equilíbrio olhos fechados - perna esquerda $(p=0,021)$, supino-flexão tempo $(\mathrm{p}=0,000)$ e prono-extensão tempo $(p=0,000)$, sendo o efeito favorável à classe média. 
Tabela 3: Efeito Isolado do nível socioeconômico no desempenho nos testes Bender, MAC, provas de equilíbrio e postura

\begin{tabular}{lccc}
\hline Variável & $\begin{array}{c}\text { Baixa renda } \\
\text { (Média } \pm \\
\text { Desvio Padrão) }\end{array}$ & $\begin{array}{c}\text { Classe Média } \\
\text { (Média } \pm \\
\text { Desvio Padrão) }\end{array}$ & $\begin{array}{c}\text { Valo p } \\
\text { (Teste de } \\
\text { Mann-Whitney) }\end{array}$ \\
\hline Bender & $9,86 \pm 4,38$ & $9,13 \pm 3,78$ & 0,394 \\
MAC acuidade - bruto & $161,02 \pm 9,35$ & $163,12 \pm 5,36$ & 0,078 \\
Equilíbrio Olhos Abertos D & $10,96 \pm 4,93$ & $14,38 \pm 1,31$ & 0,096 \\
Equilíbrio Olhos Abertos E & $10,96 \pm 4,68$ & $13,73 \pm 2,29$ & 0,071 \\
Equil. Olhos Fechados D & $5,16 \pm 4,20$ & $6,65 \pm 4,23$ & 0,028 \\
Equil. Olhos Fechados E & $4,91 \pm 3,78$ & $6,25 \pm 3,69$ & 0,022 \\
Supino-Flexão - tempo & $17,62 \pm 8,11$ & $23,18 \pm 4,05$ & 0,000 \\
Prono-Extensão - tempo & $17,66 \pm 7,97$ & $23,95 \pm 3,88$ & 0,000 \\
\hline
\end{tabular}

Tabela 4: Efeito isolado da idade gestacional no desempenho nos testes Bender, MAC, provas de equilíbrio e postura

\begin{tabular}{lcrc}
\hline Variável & $\begin{array}{c}\text { Pré-termo } \\
\text { (Média } \pm \\
\text { Desvio Padrão) }\end{array}$ & $\begin{array}{c}\text { A termo } \\
\text { (Média } \pm \\
\text { Desvio Padrão) }\end{array}$ & $\begin{array}{c}\text { Valo p } \\
\text { (Teste de } \\
\text { Mann-Whitney) }\end{array}$ \\
\hline Bender & $11,68 \pm 4,55$ & $8,59 \pm 3,60$ & 0,000 \\
MAC acuidade - bruto & $159,80 \pm 11,90$ & $162,68 \pm 5,52$ & 0,172 \\
Equilíbrio Olhos Abertos D & $14,03 \pm 8,91$ & $12,72 \pm 3,92$ & 0,114 \\
Equilíbrio Olhos Abertos E & $13,83 \pm 9,11$ & $12,54 \pm 3,74$ & 0,031 \\
Equil. Olhos Fechados D & $5,49 \pm 4,87$ & $5,84 \pm 4,24$ & 0,398 \\
Equil. Olhos Fechados E & $4,84 \pm 3,90$ & $5,63 \pm 3,76$ & 0,136 \\
Supino-Flexão - tempo & $16,23 \pm 9,01$ & $21,34 \pm 6,32$ & 0,000 \\
Prono-Extensão - tempo & $17,20 \pm 9,40$ & $21,49 \pm 6,53$ & 0,000 \\
\hline
\end{tabular}

\section{DISCUSSÃO}

Este estudo dá suporte às evidências de que crianças com história de prematuridade e/ ou de baixo nível socioeconômico têm pior desempenho em testes perceptual-motores. ${ }^{3,6,14,15,23-25}$ Ao comparar o desempenho dos três grupos, sem considerar o efeito isolado da idade gestacional e do nível socioeconômico, dentre as 10 variáveis analisadas, apenas três (MAC acuidade, equilíbrio olhos fechados perna direita, e equilíbrio olhos fechados perna esquerda) não apontaram diferença significativa de desempenho entre os grupos.
Em consonância com estudo prévio realizado no ACRIAR ${ }^{24}$, no teste MAC-acuidade, apesar do grupo pré-termo ter mostrado menor acuidade e maior variabilidade no traçado, a diferença entre os grupos não foi significativa, o que sugere que a habilidade de coordenação visomotora necessária para traçar a linha corretamente foi similar nos três grupos. No presente estudo não foi incluído o fator tempo, mas em estudos anteriores ${ }^{24,26}$ foi observado que as crianças pré-termo são mais lentas, $o$ que enfatiza a necessidade de combinar medidas de tempo e acuidade quando se examina provas de traçado. 
Observa-se, no entanto, que o desempenho das crianças pré-termo no teste Bender ${ }^{22}$, que além da coordenação viso-motora tem um componente visoespacial de cópia de figuras, foi significativamente inferior ao das crianças a termo. Tal resultado é compatível com vários estudos ${ }^{3,6,14,25,27}$ e aponta a importância do acompanhamento de crianças pré-termo no processo de escolarização, pois há evidências de que a habilidade para copiar figuras geométricas é precursora da escrita. ${ }^{28}$

Em relação às provas de equilíbrio, Magalhães et $\mathrm{a}^{20} \mathrm{e}$ Fallang et $\mathrm{a}^{29}$ apontam diferenças significativas de desempenho nas provas de equilíbrio entre crianças nascidas pré-termo e a termo. Nas provas de equilíbrio estático com olhos fechados não foram observadas diferenças significativas entre os três grupos. Nas provas de olhos abertos, foi observada diferença significativa entre os grupos pré-termo de baixa renda e a termo de classe média, e entre os grupos a termo de diferentes níveis socioeconômicos. Em ambos os casos as crianças de classe média apresentaram melhor desempenho do que as de baixa renda.

Seitz et $\mathrm{al}^{27}$ observaram pior desempenho de crianças pré-termo em provas de equilíbrio dinâmico, que não foi avaliado no presente estudo. Seria interessante, portanto, incluir a avaliação de equilíbrio dinâmico em futuros estudos sobre o desempenho psicomotor de crianças pré-termo brasileiras.

A análise do efeito isolado do nível socioeconômico apontou efeito favorável à classe média nas provas de equilíbrio de olhos abertos e de olhos fechados, para ambas as pernas, sugerindo pior desempenho para crianças de baixa renda. Como foi examinado apenas o equilíbrio estático, os resultados das provas de equilíbrio são inconclusivos, mas apontam tendência para pior desempenho das crianças em desvantagem social, pré-termo e a termo.

Nas provas posturais, os resultados apontam para influência tanto da idade gestacional quanto do nível socioeconômico no desempenho de crianças em idade escolar. Resultados semelhantes são apontados por Magalhães et $\mathrm{al}^{24} \mathrm{e}$ Foulder-Hughes e Cooke ${ }^{30}$, ao comparar o desempenho de crianças pré-termo e a termo. Não foram encontrados estudos sobre a influência do nível socioeconômico no controle postural.

De acordo com Nobre et a ${ }^{31}$ existe aceitação no meio científico de que o nível socioeconômico pode exercer influência sobre a quantidade e a qualidade dos estímulos ambientais direcionados a promover o desenvolvimento motor das crianças. $\mathrm{Na}$ amostra em estudo, as crianças da escola particular tinham acesso a quadras de esporte, piscina e aulas de educação física, o que pode ter contribuído para o melhor desempenho nas provas posturais e de equilíbrio.

Considerando que o controle postural e o equilíbrio são funções intimamente relacionadas e que dão suporte à movimentação coordenada, as crianças do grupo pré-termo têm maior risco de apresentar problemas na motricidade fina, como indicado pelo desempenho inferior no teste visomotora de Bender. Esses dados são compatíveis como os estudos de Goyen e colaboradores $^{6,32}$ revisão de Fawke ${ }^{2}$, que documentam maior frequência de transtornos menores, especialmente da coordenação motora, em crianças nascidas pré-termo e "aparentemente normais", avaliadas na idade escolar.

Embora os resultados apontem para pior desempenho motor no grupo pré-termo, esse estudo tem limitações, uma vez que a amostra não incluiu crianças pré-termo com diferentes idades gestacionais e níveis sociais.

Os dados indicam que a prematuridade associada a fatores socioeconômicos têm impacto no desenvolvimento psicomotor de crianças em idade escolar. O grupo pré-termo foi o de pior desempenho e o nível socioeconômico parece influenciar mais nas provas de equilíbrio e postura, com melhor desempenho entre as crianças de classe média. De modo geral, as diferenças observadas entre os re- 
sultados dos testes das crianças pré-termo e a termo indicam que o acompanhamento não deve se restringir aos primeiros dois anos de vida, pois essas crianças tendem a apresentar pior desempenho percepto-motor e postural na idade escolar.

Problemas percepto-motores e posturais podem ter impacto no desempenho escolar da criança, sendo importante o trabalho preventivo, executado por equipes interdisciplinares, que incluam, além do pediatra e do neurolo-

\section{REFERÊNCIAS}

1. Moster D, Lie RT, Markestad T. Long-term medical and social consequences of preterm birth. N Engl J Med. 2008; 359:262-73..

2. Fawke J. Neurological outcomes following preterm birth. Seminars in Fetal \& Neonatal Medicine. 2007; 12: 374-382.

3. Jongmans M, Mercuri E, De Vries L, Dubowitz L, Henderson S. Minor neurological signs and perceptual motor difficulties in prematurely born children. Arch Dis Child. 1997;76: F9-F14.

4. Jiménez Martín AM, Ginard CS, Jaume AR, Juan GF, Rodríguez JP. Seguimiento de recién nacidos de peso menor o igual a $1.000 \mathrm{~g}$ durante los tres primeros años de vida. Anales de Pediatría (Barcelona). 2008; 68(4): 320-328.

5. Espírito Santo JL, Portuguez MW, Nunes ML. Cognitive and behavioral status of low birth weight preterm children raised in a developing country at preschool age. Jornal de Pediatria. 2009; 35(1): 35-41.

6. Goyen T, Lui K, Woods R. Visual-motor, visual-perceptual, and fine motor outcomes in very-low-birthweight children at 5 years. Dev Med Child Neurol. 1998; 40: 76-81. gista infantil, a participação de terapeutas ocupacionais.

Assim, recomenda-se o acompanhamento do desenvolvimento de crianças prematuras até, pelo menos, a idade escolar, principalmente daquelas em situação de desvantagem social.

Agradecimento: Ao CNPq e FAPEMIG, pelo suporte ao projeto ACRIAR. À terapeuta ocupacional Renata T. Oliveira pela colaboração na coleta de dados.

7. Kleine M, den Ouden A, Kollée L, Nijhuis van der Sanden M, Sondaar M, van KesselFedema B, et al. Development and evaluation of a follow up assessment of preterm infants at 5 years of age. Arch Dis Child. 2003; 88: 870-875.

8. Fawer C, Besnier S, Forcada M, Buclin T, Calame A. Influence of perinatal, developmental and environmental factors on cognitive abilities of preterm children without major impairments at 5 years. Early Hum Devel. 1995; 43: 151-164.

9. Roth S, Baudin J, Pezzani-Goldsmith M, Townsend J, Reynolds E, Stewart A. Relation between neurodevelopmental status of very preterm infants at one and eight years. Dev Med Child Neurol. 1994; 36: 1049-1062.

10. Abreu LC, Souza AMB, Oliveira AG et al. Incidência de hemorragia peri-intraventricular em recém-nascidos pré-termo e a relação com o baixo peso ao nascer. Rev Bras Crescimento Desenvolv Hum. 2007; 17(2): 24-30.

11. Oliveira AG, Siqueira PP, Abreu LC. Cuidados nutricionais no recém-nascido de muito baixo peso. Rev Bras Crescimento Desenvolv Hum. 2008; 18(2): 148-154. 
12. Hall A, Mckeod A, Counsell C, Thomson L, Mutch L. School attainment, cognitive ability and motor function in a total scottish very-low-birthweight population at eight years: a controlled study. Dev Med Child Neurol. 1995; 37: 1037-1050.

13. Cooke RWI. Perinatal and postnatal factors in very preterm infants and subsequent cognitive and motor abilities. Arch Dis Child Fetal Neonatal Ed. 2005; 90: 60-63.

14. Saigal S. Cognitives abilities and school performance of extremely low birthweight children and matched term control children at age 8 years : a regional study. J Pediatrics. 1991; 118(5): 751-760.

15. Carvalho A, Linhares M, Martinez F. História de desenvolvimento e comportamento de crianças nascidas pré-termo e baixo peso $(<1500$ g). Psicologia: Reflexão e Crítica. 2001; 14(1): 1-33.

16. Mancini MC, Megale L, Brandão MB, Melo APP, Sampaio, RF. Estudo do desenvolvimento da função motora aos 8 e 12 meses de idade em crianças nascidas prétermo e a termo. Arq. Neuropsiquiatria. 2002; 60(4): 974-980, 2002.

17. Rugolo L. Crescimento e desenvolvimento a longo prazo do prematuro extremo. Jornal de Pediatria. 2005; 81(supl 1): S101-S110.

18. Méio M, Lopes C, Morsch D, Monteiro A, Rocha S, Borges R, et al. Desenvolvimento cognitivo de crianças prematuras de muito baixo peso na idade pré-escolar. Jornal de Pediatria. 2004; 80: 495-502.

19. Linhares M, Chimello J, Bordin M, Carvalho A, Martinez F. Desenvolvimento psicológico na fase escolar de crianças nascidas pré-termo em comparação com crianças nascidas a termo. Psicologia: Reflexão e Crítica. 2005; 18[1]: 109-117.

20. Magalhães L, Barbosa V, Lopes K, Paixão M. Estudo longitudinal do desenvolvimento de recém-nascidos pré-termo: avaliação na idade pré-escolar. Rev Bras Neurol. 1999; 35: 87-93.

21. Ayres A. Southern California Sensory Integration Tests - Revised. 1980. Los Angeles, WPS.

22. Koppitz E. Teste Gestáltico de Bender para Crianças. 1989. Porto Alegre, Artes Médicas.

23. Ross G, Lipper E, Auld P. Social competence and behavior problems in premature children at school age. Pediatrics 1991; 86: 391-397.

24. Magalhães L, Catarina P, Barbosa V, Mancini M, Paixão M. Estudo comparativo sobre o desempenho perceptual e motor na idade pré-escolar em crianças nascidas pré-termo e a termo. Arq Neuropsiquiatr. 2003; 61: 250-255.

25. Herrgard E, Luoma L, Tuppurainen K, Karjalainen S, Martikainen A. Neurodevelopmental profile at five years of children born at $<32$ weeks gestation. Dev Med Child Neurol 1993; 35: 1083-1096.

26. Carvalho DJ, Magalhães LC. A relação entre o Desenho da Figura Humana e a coordenação visomotora em crianças pré-termo aos 6 anos de idade. Revista de Terapia Ocupacional da USP. 2005. 16: 10-17.

27. Seitz J, Jenni O, Molinari L, Caflisch J, Largo R, Latal Hajnal B. Correlations between motor performance and cognitive functions in children born $<1250 \mathrm{~g}$ at school age. Neuropediatrics. 2006; 37: 6-12.

28. Daly CJ, Kelley GT, Krauss A. Relationship between visual motor integration and handwriting skills of children in kindergarten: A modified replication study. American Journal of Occupational Therapy, v.57, p.459-462, 2003.

29. Fallang B, Oien I, Hellem E, Saugstad O, Hadders-Algra M. Quality of reaching and postural control in young preterm infants 
is related to neuromotor outcome at 6 years. Pediatric Research. 2005. 58(2), 347-353.

30. Foulder-Hughes L, Cooke R. Motor, cognitive, and behavioural disorders in children born very preterm. Dev Med Child Neurol. 2003; 45: 97-103.

31. Nobre FSS, Costa CLA, Oliveira DL, Cabral DA, Nobre GC, Caçola P. Análise das oportunidades para o desenvolvimento motor (affordances) em ambientes domésticos no Ceará - Brasil. Rev Bras Crescimento Desenvolv Hum. 2009; 19(1): 9-18.

32. Goyen T-A, Lui K. Developmental coordination disorder in "apparently normal" schoolchildren born extremely preterm. Arch Dis Child. 2009; 94: 298-302.

Recebido em: 01/set./2010

Modificado em 26/set./2010 Aceito em 14/nov./2010 